\section{Priorities in health: what do they mean to Brazilian adults?}

\author{
Prioridades em saúde: o que elas significam para \\ adultos brasileiros?
}

\author{
1 Escola Superior \\ de Educação Física, \\ Universidade Federal de \\ Pelotas, Pelotas, Brasil. \\ 2 Programa de Pós-graduação \\ em Educação Física, \\ Universidade Federal de \\ Pelotas, Pelotas, Brasil. \\ 3 Programa de Pós-graduação \\ em Epidemiologia, \\ Universidade Federal de \\ Pelotas, Pelotas, Brasil. \\ 4 Centro de Ciências da Vida \\ e da Saúde, Universidade \\ Católica de Pelotas, Pelotas, \\ Brasil. \\ Correspondence \\ F. F. Reichert \\ Escola Superior de Educação \\ Física, Universidade Federal \\ de Pelotas. \\ Rua Luiz de Camões 625, \\ Pelotas, RS 96055-630, Brasil. \\ ffreichert@gmail.com
}

\begin{abstract}
We aimed to evaluate how Brazilian adults rank seven well-known health-related factors in terms of importance for health. A population-based study was undertaken in Pelotas, Rio Grande do Sul State, Brazil ( $N=3,100$; response rate: $96.5 \%)$. Individuals ranked three out of seven factors that, in their opinion, were the most important for health. The factors investigated were: "controlling stress", "practicing physical activity regularly", "avoiding drinking in excess", "avoiding smoking", "visiting a doctor regularly", "keeping the ideal weight", and "having a healthy diet". Healthy diet (73.9\%), physical activity (59.9\%), and visiting a doctor regularly (45.7\%) were the most frequently reported factors. Younger subjects and those with higher socioeconomic status were more likely to report physical activity and stress as important factors for health than their counterparts. The importance attributed to health-related factors changes markedly among population subgroups.
\end{abstract}

Adult Health; Risk Factors; Diet; Motor Activity
Felipe Fossati Reichert 1

Marlos Rodrigues Domingues 1,2

Pedro C. Hallal 1,2,3

Mario Renato Azevedo 1

Fernando Vinholes Siqueira 4

Aluísio J.D. Barros 3

\section{Introduction}

Because of the epidemiological transition, risk factors for chronic diseases are getting widespread attention in the scientific literature. Since the 1950s, the harmful effects of smoking and physical inactivity on the risk of lung cancer and coronary disease, respectively, have been discussed 1,2. More recently, abusive alcohol consumption, and obesity were considered to be risk factors for several chronic diseases $3,4,5,6,7,8$. In addition, there is growing evidence suggesting that stress is an underlying risk factor for many conditions 4,9 .

Although several studies have investigated the prevalence of such exposures 10,11,12,13, and others have evaluated population knowledge on the importance of such factors for health 14,15,16, only a few have stimulated individuals to rank such factors with respect to their relevance 17 . From a public health perspective, this information is important because it might reflect how scientific knowledge is reaching the population.

In the European Union, 15,239 individuals aged 15 years or older were asked to select two, out of eight factors, which, according to their beliefs, had the greatest influence on overall health 17. The factors studied and their rank were: smoking (chosen by $41 \%$ of individuals), food $(38 \%)$, stress $(33 \%)$, alcohol intake $(20 \%)$, physical activity (18\%), environment (16\%), body weight (13\%), and genetics and metabo- 
lism (9\%). Nonetheless, there was a wide intercountry variation in the rank of the factors studied. For example, physical activity was selected by $44 \%$ of respondents in Finland, and only $9 \%$ in Greece and Italy 17 . The identification and understanding of the reasons for such marked differences between countries and between population subgroups is an important step in tackling the high rates of chronic disease worldwide.

Currently there is no similar data from lowand middle-income countries, and it is quite possible that the epidemiology of these data differ from richer settings. Therefore, a populationbased study with Brazilian adults was carried out with the primary aim of describing the population rank of importance to overall health of seven well-known health-related factors.

\section{Materials and methods}

A health survey was carried out in Pelotas, a medium-sized Southern Brazilian city $(\sim 320,000$ inhabitants) in the second semester of 2003. In Pelotas, the prevalence of obesity (body mass index - BMI $\geq 30 \mathrm{~kg} . \mathrm{m}^{-2}$ ) in adults is around $15 \%$; the prevalence of physical inactivity is above $40 \%$; and the prevalence of smoking is nearly $30 \% 12$. In terms of alcohol use, the prevalence of heavy alcohol consumption (above $30 \mathrm{~g} /$ day) is $14 \% 11$.

The present survey was based on a two-stage clustered sample. The primary sample units were the census tracts delimited by the Brazilian Institute of Geography and Statistics in 2000 (IBGE; http://www.ibge.gov.br). Each of these tracts comprises approximately 300 households. After stratification for the average income of family heads, 144 census tracts were sampled with probability proportional to the cluster size. Households were selected within each census tract by systematic sampling. All residents aged 20 years or older were eligible for this investigation.

Information on the importance for health of the factors studied was gathered as follows: a card containing seven pictures of relevant aspects of health was shown to subjects. Each image was presented along with a phrase indicating its exact meaning: "controlling stress", "practicing physical activity regularly", "avoiding drinking in excess", "avoiding smoking", "visiting a doctor regularly", "keeping the ideal weight”, and "having a healthy diet". Individuals were asked to rank the three factors that, in their opinion, were the most relevant for health. These factors were chosen based on the single study available about this subject 17 while also taking into consideration cultural aspects of Brazilian people.
Behavioral factors were preferred because they are modifiable.

A standardized and pre-tested questionnaire was used to collect all data. The study is part of a large survey and several other health-related outcomes were investigated (physical activity, medicine consumption, health services and campaigns, knowledge on contraceptive methods, migraine, and symptoms of several morbidities). Hence, the question about the health indicators was placed at the beginning of the questionnaire, before the investigation of the other health outcomes, in an attempt to avoid the potential influence of previous questions over the answers.

The independent variables studied were: sex, age, skin color (as observed by the interviewer), wealth status (using the Brazilian Criteria for Economic Classification, an asset-based evaluation that considers also the schooling of the family head; and where A is the wealthiest group; http://www.abep.org), schooling (highest degree completed), BMI (based on self-reported weight and height), smoking (smokers were defined as those who smoked at least one cigarette per day in the last 30 days) and leisure-time physical activity level. Physical activity was evaluated using the leisure-time section of the long version of the International Physical Activity Questionnaire (IPAQ). A physical activity score was calculated as the sum of the weekly minutes spent in moderate activities (including walking) plus twice the weekly time spent in vigorous activities 12 . Individuals with score zero were considered "sedentary", those with scores between 10 and 149 were classified as "insufficiently active" and those with score $\geq 150$ were considered "sufficiently active", in accordance with current physical activity recommendations 18 .

Data were collected during face-to-face home-based interviews. The interviewers were all women with at least high school education who underwent 40 hours of training.

Analyses were performed using Stata 8.2 (Stata Corp., College Station, U.S.A.) and took into consideration the clustering of the sample (by using the "survey" group of commands). Wald tests for heterogeneity were used for non-ordinal categorical exposures, while tests for linear trend were used for ordinal exposures. Multivariable analyses were carried out using Poisson regression 19. A hierarchical causation model was previously established. The model included demographic variables at distal level (sex, age and skin color), socioeconomic variables at intermediate level (socioeconomic level and schooling), and smoking, BMI, and leisure-time physical activity at the proximal level. The variables were entered 
into the model by level, and only those with a pvalue $\leq 0.20$ were kept in the regression.

The Ethics Committee of the Medical School of the Federal University of Pelotas approved the research protocol, and informed consent was obtained from each subject before the interview.

\section{Results}

From the 3,214 eligible individuals, 3,100 were interviewed (non-response rate: $3.5 \%$ ). Most individuals were white $(81 \%)$ and more than half of the sample was female (56.7\%). Mean age and schooling were $43.2(\mathrm{SD}=16.1)$ and $7.7(\mathrm{SD}=4.4)$ years, respectively. In terms of behavioral variables, $26.7 \%$ of the subjects were current smokers and $58.1 \%$ were sedentary; only $26.8 \%$ achieved the cutoff point to be considered sufficiently active. The prevalence of overweight (BMI $\geq 25.0$ or $\leq 29.9 \mathrm{~kg} . \mathrm{m}^{-2}$ ) and obesity (BMI $\geq 30 \mathrm{~kg} . \mathrm{m}^{-2}$ ) were $33.3 \%$ and $13.8 \%$, respectively.

Table 1 presents the proportion of individuals reporting each of the health indicators studied, regardless of ranking. Having a healthy diet and engaging in physical activity were the most reported factors $(73.9 \%$ and $59.9 \%$, respectively). Minor changes in the rank of health indicators were observed when considering only the individuals' first choice (Figure 1). For example, although, as a whole, the proportion of physical activity was much higher than reporting on avoiding smoking, when considering only the first factor mentioned, their values are comparable. Having a healthy diet was again the most frequently reported factor.

The association between the health indicator chosen (regardless of ranking order) and the independent variables is also shown in Table 1. Women more frequently reported that having a healthy diet is important for health. In contrast, men cited physical activity as an important factor for health more frequently than women. The direction of the association between age and the health indicators varied. For example, the younger the person, the more likely that he/she would report physical activity as an important factor for health, whilst the less likely that he/she would report avoiding drinking in excess. Obese individuals reported keeping the ideal weight $55 \%$ more frequently than those with $\mathrm{BMI}<18.5 \mathrm{~kg} \cdot \mathrm{m}^{-2}$. A similar pattern was identified between smoking behavior and reporting avoiding smoking as an important determinant of health (current and former smokers reported it more frequently than never smokers). However, a distinct association was presented with the health indicator physical activity: sedentary individuals reported physical activity as an important factor for health less frequently than active ones.

The markedly different association between wealth status and two health indicators, physical activity and avoiding smoking, is illustrated in Figure 2. Both socioeconomic variables (wealth status and schooling) showed similar associations with most health indicators. The higher the level of schooling, the higher the proportion of reports for having a healthy diet, engaging in physical activities, and controlling stress as major health determinants. The inverse was observed with the remaining health indicators.

Table 2 shows the association between the first mentioned health indicator (the most important in influencing overall health, according to individuals' opinions) and the independent variables. Overall, the association between the variables followed the pattern observed in Table 1. However, minor differences were also noted. For example, no association between skin color and reporting physical activity was observed $(\mathrm{p}=$ 0.72). Furthermore, the trends between age and the health-related factors physical activity and keeping the ideal weight observed in Table 1, were not observed in Table 2. On the other hand, a positive trend between BMI and the healthrelated factor avoiding drinking in excess was observed ( $\mathrm{p}=0.02)$.

We also carried out multivariable analyses and the results were similar to those obtained during crude analysis, as presented in Table 3.

\section{Discussion}

This study brings information from a middleincome country, where data on health information and knowledge are often lacking. Usually, data from high-income countries lend support to public health interventions in poorer countries, despite marked differences in several health aspects between these settings.

The sampling process and the high response rate $(96.5 \%)$ highlight the methodological care of the study and allow us to rule out the possibility of selection bias with great confidence. Furthermore, the investigation of other health outcomes in the present survey did not influence our results because the question on the importance of the health indicators was placed at the beginning of the questionnaire. However, some limitations of the study must be discussed: we do not know to what extent the chosen factors (and images) are reliable for representing health relationships in our population, although we have tested extensively the methodology employed and did not observe any understanding issues. 
Table 1

Prevalence and associated factors of the perceived health indicators, regardless of the order of citation. Pelotas, Rio Grande do Sul State, Brazil, 2003 $(\mathrm{N}=3,100)$.

\begin{tabular}{|c|c|c|c|c|c|c|c|}
\hline Variables & Healthy diet & $\begin{array}{l}\text { Physical } \\
\text { activity }\end{array}$ & $\begin{array}{l}\text { Seeing a } \\
\text { doctor }\end{array}$ & Not smoking & $\begin{array}{l}\text { Not drinking } \\
\text { alcohol in } \\
\text { excess }\end{array}$ & $\begin{array}{l}\text { Avoiding } \\
\text { stress }\end{array}$ & $\begin{array}{l}\text { Keeping the } \\
\text { ideal weight }\end{array}$ \\
\hline Overall & 73.9 & 59.9 & 45.7 & 45.5 & 31.8 & 25.1 & 18.2 \\
\hline Sex & $p<0.001$ * & $p<0.001$ * & $p=0.55$ * & $p=0.90$ * & $p=0.53$ * & $p=0.29$ * & $p=0.79 *$ \\
\hline Male & 69.8 & 64.5 & 45.1 & 45.6 & 32.3 & 24.2 & 18.4 \\
\hline Female & 77.0 & 56.3 & 46.2 & 45.4 & 31.4 & 25.7 & 18.1 \\
\hline Skin color & $p=0.01$ * & $p=0.02$ * & $p<0.001$ * & $p=0.25$ * & $p=0.01$ * & $p=0.61$ * & $p=0.94$ * \\
\hline White & 74.9 & 61.1 & 43.9 & 45.9 & 30.8 & 25.3 & 18.2 \\
\hline Non-white & 69.6 & 54.8 & 53.6 & 43.5 & 36.2 & 24.2 & 18.1 \\
\hline Age (years) & $p=0.33 * \star$ & $p<0.001$ ** & $p=0.31 \star \star$ & $p=0.67 * \star$ & $p<0.001 * \star$ & $p=0.27^{\star \star}$ & $p<0.001$ ** \\
\hline $20-29$ & 72.2 & 69.0 & 44.8 & 49.0 & 28.2 & 23.3 & 13.4 \\
\hline $30-49$ & 74.0 & 60.7 & 45.3 & 43.1 & 30.3 & 27.2 & 19.4 \\
\hline $50-59$ & 75.4 & 55.7 & 47.2 & 45.9 & 33.3 & 25.6 & 16.9 \\
\hline $60-69$ & 72.8 & 50.0 & 44.9 & 44.9 & 40.4 & 23.2 & 23.9 \\
\hline$\geq 70$ & 76.6 & 46.0 & 49.0 & 47.3 & 38.1 & 20.1 & 23.0 \\
\hline Wealth status & $p<0.001 \star \star$ & $p<0.001 \star \star$ & $p<0.001 * \star$ & $p<0.001 \star \star$ & $p<0.001 * \star$ & $p=0.03 * \star$ & $p=0.59 * \star$ \\
\hline$A / B$ & 78.2 & 70.7 & 41.6 & 39.2 & 22.6 & 29.5 & 18.4 \\
\hline C & 76.1 & 62.9 & 44.9 & 44.7 & 30.3 & 23.4 & 17.7 \\
\hline D & 70.9 & 51.9 & 47.5 & 49.5 & 38.3 & 24.0 & 17.8 \\
\hline$E$ & 62.9 & 43.7 & 54.8 & 53.3 & 40.6 & 22.3 & 22.3 \\
\hline Schooling (years) & $p<0.001 \star \star$ & $p<0.001 * \star$ & $p<0.001 * \star$ & $p=0.01 \star \star$ & $p<0.001 * \star$ & $p<0.001 * \star$ & $p=0.02 * \star$ \\
\hline$\geq 12$ & 82.1 & 73.8 & 38.0 & 37.6 & 16.2 & 33.8 & 18.6 \\
\hline $9-11$ & 75.3 & 66.5 & 43.1 & 45.6 & 28.2 & 27.0 & 14.3 \\
\hline $5-8$ & 71.0 & 57.4 & 48.9 & 48.4 & 35.1 & 21.0 & 18.3 \\
\hline $0-4$ & 71.6 & 48.1 & 48.8 & 46.2 & 40.1 & 23.3 & 21.9 \\
\hline Body mass index $(\mathrm{kg} \cdot \mathrm{m}-2)$ & $p=0.48 * \star$ & $p=0.27 \star \star$ & $p=0.52 \star \star$ & $p=0.24 * \star$ & $p=0.30 * \star$ & $p=0.93 * \star$ & $\mathrm{p}<0.001$ ** \\
\hline$<18.5$ & 79.1 & 56.7 & 44.8 & 44.8 & 32.8 & 25.4 & 16.4 \\
\hline $18.5-24.9$ & 74.1 & 62.7 & 44.4 & 46.0 & 32.3 & 25.1 & 15.4 \\
\hline $25.0-29.9$ & 73.0 & 61.5 & 44.0 & 46.1 & 30.1 & 25.9 & 19.5 \\
\hline$\geq 30.0$ & 73.8 & 57.7 & 47.3 & 41.0 & 30.1 & 24.9 & 25.4 \\
\hline Smoking & $p<0.001$ * & $p=0.002$ * & $p=0.85$ * & $p=0.006$ * & $p<0.001$ * & $p=0.13$ * & $p=0.70$ * \\
\hline Never smoked & 78.2 & 63.1 & 45.8 & 42.6 & 28.6 & 24.0 & 17.7 \\
\hline Former smoker & 70.1 & 56.6 & 44.8 & 47.4 & 35.1 & 28.1 & 18.1 \\
\hline Current smoker & 68.8 & 56.5 & 46.3 & 49.6 & 35.0 & 24.7 & 19.2 \\
\hline Physical activity level (min.wk-1) & $p=0.92 * \star$ & $p<0.001 * \star$ & $p=0.005^{\star *}$ & $p=0.06 * \star$ & $p=0.001 * \star$ & $p=0.15^{\star \star}$ & $p=0.66 * \star$ \\
\hline 0 & 73.5 & 52.2 & 47.3 & 47.8 & 34.7 & 25.8 & 18.7 \\
\hline $10-149$ & 76.5 & 65.5 & 49.7 & 38.5 & 28.1 & 25.3 & 16.5 \\
\hline$\geq 150$ & 73.4 & 73.1 & 40.0 & 44.6 & 27.6 & 23.1 & 18.3 \\
\hline
\end{tabular}

* Wald test for heterogeneity;

** Wald test for trend.

Subjects were asked to rank three out of seven well known health-related factors in terms of importance for health. Important differences were observed among the first, second and third health indicator cited. The three most frequently cited factors were having a healthy diet, practic- ing physical activity and visiting a doctor regularly, respectively. By using this strategy, we tried to understand how the population perceives the risks that these factors pose to overall health. We are aware that all the factors addressed in the present study are important, and answering our 
Figure 1

Health indicators according to the rank they were perceived. Pelotas, Rio Grande do Sul State, Brazil, $2003(N=3,100)$.

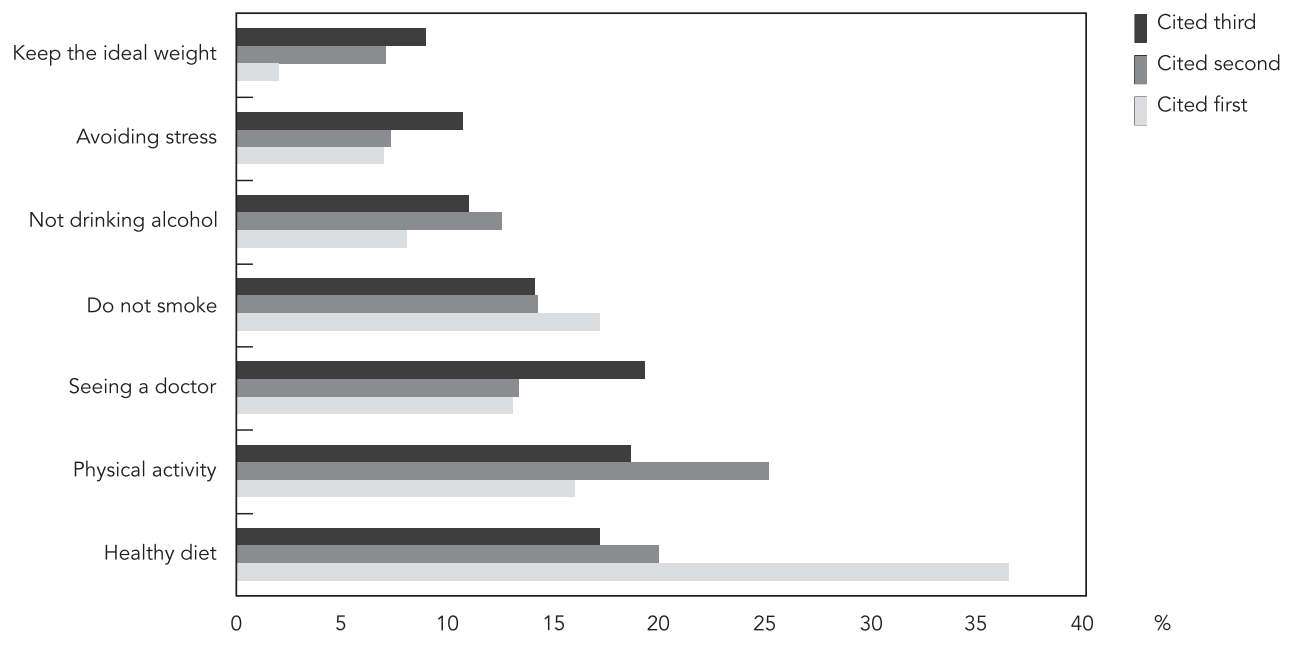

Figure 2

Perception of physical activity and avoiding smoking according to wealth status (regardless of the order they were cited). Pelotas, Rio Grande do Sul State, Brazil, 2003 (N = 3,100).

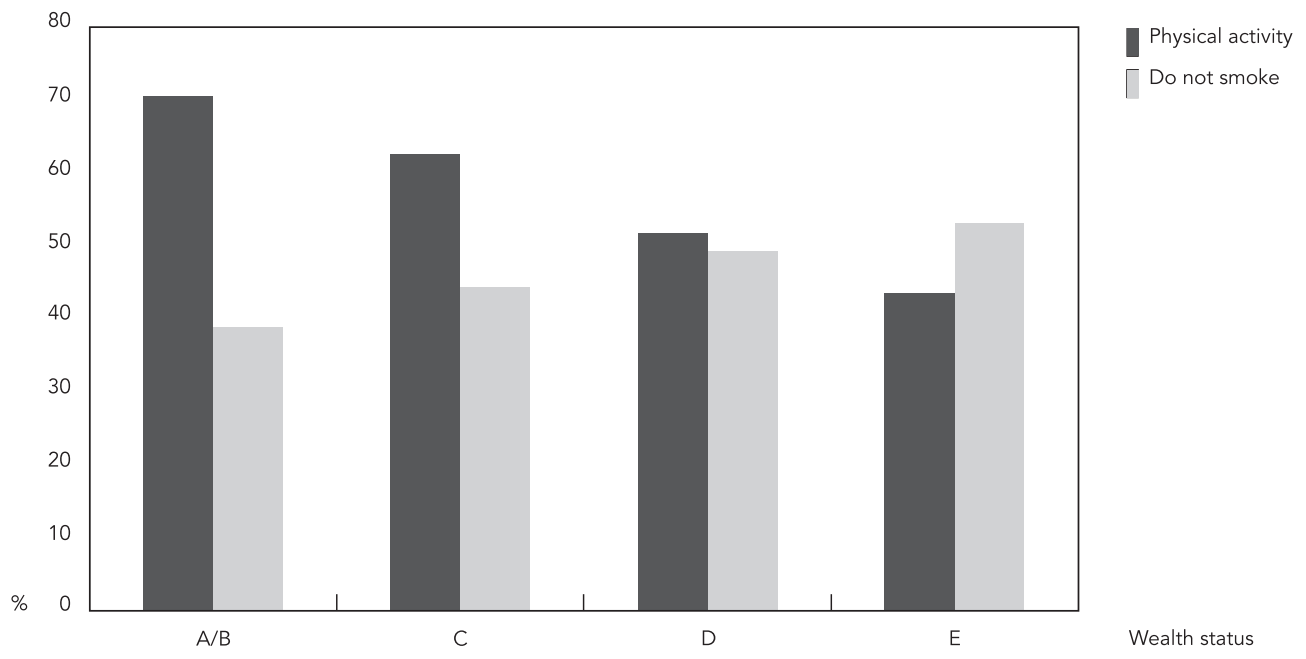


Table 2

First health indicator cited and its associated factors. Pelotas, Rio Grande do Sul State, Brazil, $2003(N=3,100)$.

\begin{tabular}{|c|c|c|c|c|c|c|c|}
\hline Variables & Healthy diet & $\begin{array}{l}\text { Physical } \\
\text { Activity }\end{array}$ & $\begin{array}{l}\text { Seeing a } \\
\text { doctor }\end{array}$ & Not smoking & $\begin{array}{l}\text { Not drinking } \\
\text { alcohol in } \\
\text { excess }\end{array}$ & $\begin{array}{l}\text { Avoiding } \\
\text { stress }\end{array}$ & $\begin{array}{l}\text { Keeping the } \\
\text { ideal weight }\end{array}$ \\
\hline Overall & 36.5 & 16.0 & 13.1 & 17.2 & 8.1 & 7.0 & 2.1 \\
\hline Sex & $p<0.001$ * & $p<0.001$ * & $p=0.47$ * & $p=0.35$ * & $p=0.08$ * & $p=0.65$ * & $p=0.49$ * \\
\hline Male & 31.2 & 19.5 & 13.6 & 17.9 & 9.2 & 6.8 & 1.9 \\
\hline Female & 40.6 & 13.4 & 12.7 & 16.6 & 7.3 & 7.2 & 2.2 \\
\hline Skin color & $p=0.01 *$ & $p=0.72$ * & $p<0.001$ * & $p=0.47$ * & $p=0.004$ * & $p=0.62$ * & $p=0.30$ * \\
\hline White & 38.3 & 16.1 & 12.0 & 16.9 & 7.4 & 7.1 & 2.2 \\
\hline Non-white & 29.0 & 15.5 & 17.9 & 18.3 & 11.3 & 6.5 & 1.5 \\
\hline Age (years) & $p=0.42 \star \star *$ & $p=0.17^{\star \star}$ & $p=0.68 * \star$ & $0=0.44^{\star \star}$ & $p=0.005^{\star \star}$ & $p=0.27 \star \star$ & $p=0.39 * \star$ \\
\hline $20-29$ & 33.3 & 18.8 & 13.8 & 19.4 & 5.7 & 6.9 & 2.1 \\
\hline $30-49$ & 38.4 & 15.0 & 12.9 & 16.4 & 8.3 & 7.3 & 1.7 \\
\hline $50-59$ & 36.2 & 15.2 & 12.6 & 16.3 & 8.9 & 8.5 & 2.2 \\
\hline $60-69$ & 36.0 & 15.1 & 12.9 & 15.4 & 11.8 & 5.5 & 3.3 \\
\hline$\geq 70$ & 37.7 & 15.1 & 13.0 & 18.4 & 9.2 & 4.6 & 2.1 \\
\hline Wealth status & $p=0.002 * *$ & $p=0.03^{\star \star}$ & $p<0.001$ ** & $p=0.007 * \star$ & $p=0.002 * \star$ & $p=0.007 * *$ & $p=0.16$ ** \\
\hline$A / B$ & 39.3 & 18.5 & 10.3 & 14.5 & 5.7 & 10.3 & 1.4 \\
\hline C & 40.5 & 16.0 & 11.4 & 16.4 & 7.3 & 5.8 & 2.6 \\
\hline $\mathrm{D}$ & 32.2 & 14.9 & 15.7 & 19.4 & 10.3 & 6.0 & 1.6 \\
\hline$E$ & 30.5 & 12.2 & 17.3 & 19.8 & 10.2 & 5.1 & 5.1 \\
\hline Schooling (years) & $p=0.001 \star \star *$ & $p=0.34^{\star \star}$ & $p=0.001$ ** & $p=0.12 \star \star$ & $p<0.001$ ** & $p<0.001$ ** & $p=0.009 * \star$ \\
\hline$\geq 12$ & 44.3 & 17.5 & 8.1 & 13.8 & 3.1 & 12.0 & 1.3 \\
\hline $9-11$ & 37.9 & 16.4 & 12.4 & 16.9 & 6.3 & 9.0 & 1.2 \\
\hline $5-8$ & 34.9 & 15.8 & 15.1 & 18.3 & 9.2 & 4.6 & 2.2 \\
\hline $0-4$ & 32.8 & 15.0 & 14.2 & 17.9 & 11.7 & 5.1 & 3.3 \\
\hline Body mass index (kg.m-2) & $\mathrm{p}=0.31 \star \star$ & $p=0.96 \star \star$ & $p=0.49 \star \star$ & $p=0.06 * \star$ & $p=0.02 * \star$ & $\mathrm{p}=0.51 \star \star$ & $p=0.40 * \star$ \\
\hline$<18.5$ & 47.8 & 11.9 & 9.0 & 22.4 & 4.5 & 3.0 & 1.5 \\
\hline $18.5-24.9$ & 36.5 & 16.9 & 12.8 & 18.0 & 7.2 & 6.9 & 1.9 \\
\hline $25.0-29.9$ & 36.7 & 17.1 & 11.9 & 16.4 & 8.5 & 7.8 & 1.6 \\
\hline$\geq 30.0$ & 34.7 & 15.6 & 14.5 & 15.0 & 10.4 & 6.8 & 3.0 \\
\hline Smoking & $p<0.001 *$ & $p=0.40$ * & $p=0.06$ * & $p=0.001$ * & $p=0.002$ * & $p=0.47$ * & $p=0.29$ * \\
\hline Never smoked & 40.3 & 16.8 & 12.7 & 15.0 & 6.4 & 6.5 & 2.4 \\
\hline Former smoker & 34.6 & 15.5 & 11.2 & 20.3 & 10.2 & 7.7 & 1.5 \\
\hline Current smoker & 31.0 & 15.0 & 15.4 & 18.8 & 9.6 & 7.4 & 1.9 \\
\hline Physical activity level (min.wk-1) & $p=0.18 * \star$ & $\mathrm{p}<0.001 * \star$ & $p=0.21 \star \star$ & $p=0.17^{\star \star}$ & $p=0.07 \star \star$ & $p=0.26 * \star$ & $\mathrm{p}=0.89 \star \star$ \\
\hline 0 & 37.1 & 12.2 & 13.3 & 18.3 & 9.1 & 7.8 & 2.2 \\
\hline $10-149$ & 40.9 & 16.3 & 15.6 & 14.1 & 6.6 & 4.9 & 1.5 \\
\hline$\geq 150$ & 33.2 & 23.9 & 10.9 & 16.5 & 6.9 & 6.5 & 2.2 \\
\hline
\end{tabular}

* Wald test for heterogeneity;

** Wald test for trend.

questionnaire is a challenge even for health professionals. We also recognize that self-perception issues do influence decisions. For example, people exposed to highly demanding jobs are more likely to consider stress as a major risk factor.

The understanding of the reasons that lead somebody to chose factor A or B is rather com- plex. Because all health indicators studied are largely known by the population, the knowledge about the effects of an indicator should not be considered the only factor that determines individuals' choices. It has been previously demonstrated that this sort of decision may be motivated and influenced by social and cultural aspects 
Multivariable analyses of the association between outcomes (health indicators) and exposures. Pelotas, Rio Grande do Sul State, Brazil, 2003 ( $N=3,100$ )

\begin{tabular}{|c|c|c|c|c|c|c|c|}
\hline Variables & Healthy diet & $\begin{array}{l}\text { Physical } \\
\text { activity }\end{array}$ & $\begin{array}{c}\text { Seeing a } \\
\text { doctor }\end{array}$ & Not smoking & $\begin{array}{l}\text { Not drinking } \\
\text { alcohol in } \\
\text { excess }\end{array}$ & $\begin{array}{l}\text { Avoiding } \\
\text { stress }\end{array}$ & $\begin{array}{l}\text { Keeping the } \\
\text { ideal weight }\end{array}$ \\
\hline & PR $(95 \% \mathrm{Cl})$ & PR $(95 \% \mathrm{Cl})$ & PR $(95 \% \mathrm{Cl})$ & PR (95\%Cl) & PR $(95 \% \mathrm{Cl})$ & PR $(95 \% \mathrm{Cl})$ & PR $(95 \% \mathrm{Cl})$ \\
\hline Sex & $p<0.001$ * & $p<0.001$ * & $p=0.53$ * & $p=0.91$ * & $p=0.48$ * & $p=0.32$ * & $p=0.69$ * \\
\hline Male & 1.00 & 1.00 & 1.00 & 1.00 & 1.00 & 1.00 & 1.00 \\
\hline Female & $\begin{array}{c}1.10 \\
(1.06 ; 1.15)\end{array}$ & $\begin{array}{c}0.88 \\
(0.83 ; 0.93)\end{array}$ & $\begin{array}{c}1.03 \\
(0.95 ; 1.11)\end{array}$ & $\begin{array}{c}1.00 \\
(0.92 ; 1.08)\end{array}$ & $\begin{array}{c}0.96 \\
(0.87 ; 1.07)\end{array}$ & $\begin{array}{c}1.07 \\
(0.94 ; 1.21)\end{array}$ & $\begin{array}{c}0.97 \\
(0.83 ; 1.22)\end{array}$ \\
\hline Skin color & $p=0.02$ * & $p=0.03$ * & $p<0.001$ * & $p=0.30$ * & $p=0.005$ * & $p=0.59$ * & $p=0.95$ * \\
\hline White & 1.00 & 1.00 & 1.00 & 1.00 & 1.00 & 1.00 & 1.00 \\
\hline Non-white & $\begin{array}{c}0.93 \\
(0.88 ; 0.99)\end{array}$ & $\begin{array}{c}0.89 \\
(0.82 ; 0.96)\end{array}$ & $\begin{array}{c}1.22 \\
(1.12 ; 1.33)\end{array}$ & $\begin{array}{c}0.95 \\
(0.86 ; 1.05)\end{array}$ & $\begin{array}{c}1.19 \\
(1.05 ; 1.34)\end{array}$ & $\begin{array}{c}0.96 \\
(0.82 ; 1.12)\end{array}$ & $\begin{array}{c}1.01 \\
(0.83 ; 1.22)\end{array}$ \\
\hline Age (years) & $p=0.35$ ** & $p<0.001$ ** & $p=0.24$ ** & $p=0.61 \star \star$ & $p<0.001$ ** & $p=0.27$ ** & $p<0.001$ ** \\
\hline $20-29$ & 1.00 & 1.00 & 1.00 & 1.00 & 1.00 & 1.00 & 1.00 \\
\hline $30-49$ & $\begin{array}{c}1.03 \\
(0.97 ; 1.08)\end{array}$ & $\begin{array}{c}0.88 \\
(0.83 ; 0.94)\end{array}$ & $\begin{array}{c}1.00 \\
(0.91 ; 1.11)\end{array}$ & $\begin{array}{c}0.88 \\
(0.80 ; 0.97)\end{array}$ & $\begin{array}{c}1.07 \\
(0.93 ; 1.23)\end{array}$ & $\begin{array}{c}1.17 \\
(1.00 ; 1.36)\end{array}$ & $\begin{array}{c}1.44 \\
(1.17 ; 1.78)\end{array}$ \\
\hline $50-59$ & $\begin{array}{c}1.04 \\
(0.97 ; 1.11)\end{array}$ & $\begin{array}{c}0.81 \\
(0.74 ; 0.88)\end{array}$ & $\begin{array}{c}1.06 \\
(0.94 ; 1.20)\end{array}$ & $\begin{array}{c}0.94 \\
(0.83 ; 1.06)\end{array}$ & $\begin{array}{c}1.19 \\
(1.00 ; 1.41)\end{array}$ & $\begin{array}{c}1.10 \\
(0.90 ; 1.34)\end{array}$ & $\begin{array}{c}1.26 \\
(0.96 ; 1.64)\end{array}$ \\
\hline $60-69$ & $\begin{array}{c}1.00 \\
(0.92 ; 1.09)\end{array}$ & $\begin{array}{c}0.72 \\
(0.64 ; 0.82)\end{array}$ & $\begin{array}{c}1.01 \\
(0.86 ; 1.17)\end{array}$ & $\begin{array}{c}0.91 \\
(0.79 ; 1.06)\end{array}$ & $\begin{array}{c}1.44 \\
(1.20 ; 1.73)\end{array}$ & $\begin{array}{c}0.99 \\
(0.77 ; 1.28)\end{array}$ & $\begin{array}{c}1.78 \\
(1.35 ; 2.35)\end{array}$ \\
\hline$\geq 70$ & $\begin{array}{c}1.05 \\
(0.97 ; 1.14)\end{array}$ & $\begin{array}{c}0.67 \\
(0.58 ; 0.78)\end{array}$ & $\begin{array}{c}1.10 \\
(0.95 ; 1.28)\end{array}$ & $\begin{array}{c}0.96 \\
(0.83 ; 1.12)\end{array}$ & $\begin{array}{c}1.36 \\
(1.11 ; 1.66)\end{array}$ & $\begin{array}{c}0.86 \\
(0.64 ; 1.14)\end{array}$ & $\begin{array}{c}1.71 \\
(1.28 ; 2.30)\end{array}$ \\
\hline Wealth status & $p=0.004$ ** & $p<0.001$ ** & $p=0.42$ ** & $p<0.001$ ** & $p=0.003 * \star$ & $p=0.97 * *$ & $p=0.57 * \star$ \\
\hline$A / B$ & 1.00 & 1.00 & 1.00 & 1.00 & 1.00 & 1.00 & 1.00 \\
\hline C & $\begin{array}{c}0.99 \\
(0.94 ; 1.05)\end{array}$ & $\begin{array}{c}0.94 \\
(0.87 ; 1.01)\end{array}$ & $\begin{array}{c}1.01 \\
(0.90 ; 1.13)\end{array}$ & $\begin{array}{c}1.14 \\
(1.02 ; 1.28)\end{array}$ & $\begin{array}{c}1.17 \\
(0.99 ; 1.39)\end{array}$ & $\begin{array}{c}0.88 \\
(0.75 ; 1.04)\end{array}$ & $\begin{array}{c}0.91 \\
(0.73 ; 1.12)\end{array}$ \\
\hline $\mathrm{D}$ & $\begin{array}{c}0.94 \\
(0.88 ; 1.00)\end{array}$ & $\begin{array}{c}0.81 \\
(0.74 ; 0.89)\end{array}$ & $\begin{array}{c}1.02 \\
(0.90 ; 1.15)\end{array}$ & $\begin{array}{c}1.27 \\
(1.14 ; 1.41)\end{array}$ & $\begin{array}{c}1.33 \\
(1.11 ; 1.60)\end{array}$ & $\begin{array}{c}0.98 \\
(0.82 ; 1.17)\end{array}$ & $\begin{array}{c}0.86 \\
(0.68 ; 1.08)\end{array}$ \\
\hline $\mathrm{E}$ & $\begin{array}{c}0.85 \\
(0.75 ; 0.96)\end{array}$ & $\begin{array}{c}0.70 \\
(0.58 ; 0.84)\end{array}$ & $\begin{array}{c}1.11 \\
(0.93 ; 1.33)\end{array}$ & $\begin{array}{c}1.36 \\
(1.16 ; 1.59)\end{array}$ & $\begin{array}{c}1.31 \\
(1.02 ; 1.68)\end{array}$ & $\begin{array}{c}0.97 \\
(0.71 ; 1.33)\end{array}$ & $\begin{array}{c}1.04 \\
(0.74 ; 1.46)\end{array}$ \\
\hline Schooling (years) & $p=0.14 * \star$ & $p=0.001 * \star$ & $p=0.001 * *$ & $p=0.99 * \star$ & $p<0.001$ ** & $p<0.001$ ** & $p=0.14 * \star$ \\
\hline$\geq 12$ & 1.00 & 1.00 & 1.00 & 1.00 & 1.00 & 1.00 & 1.00 \\
\hline $9-11$ & $\begin{array}{c}0.95 \\
(0.89 ; 1.01)\end{array}$ & $\begin{array}{c}0.95 \\
(0.88 ; 1.02)\end{array}$ & $\begin{array}{c}1.11 \\
(0.97 ; 1.28)\end{array}$ & $\begin{array}{c}1.14 \\
(0.99 ; 1.32)\end{array}$ & $\begin{array}{c}1.64 \\
(1.29 ; 2.09)\end{array}$ & $\begin{array}{c}0.80 \\
(0.67 ; 0.94)\end{array}$ & $\begin{array}{c}0.78 \\
(0.61 ; 1.01)\end{array}$ \\
\hline $5-8$ & $\begin{array}{c}0.92 \\
(0.86 ; 0.98)\end{array}$ & $\begin{array}{c}0.89 \\
(0.82 ; 0.97)\end{array}$ & $\begin{array}{c}1.25 \\
(1.10 ; 1.43)\end{array}$ & $\begin{array}{c}1.15 \\
(0.99 ; 1.33)\end{array}$ & $\begin{array}{c}1.89 \\
(1.49 ; 2.41)\end{array}$ & $\begin{array}{c}0.62 \\
(0.52 ; 0.74)\end{array}$ & $\begin{array}{c}0.96 \\
(0.76 ; 1.21)\end{array}$ \\
\hline $0-4$ & $\begin{array}{c}0.94 \\
(0.88 ; 1.02)\end{array}$ & $\begin{array}{c}0.83 \\
(0.74 ; 0.93)\end{array}$ & $\begin{array}{c}1.24 \\
(1.08 ; 1.42)\end{array}$ & $\begin{array}{c}1.05 \\
(0.89 ; 1.23)\end{array}$ & $\begin{array}{c}1.98 \\
(1.53 ; 2.57)\end{array}$ & $\begin{array}{c}0.69 \\
(0.58 ; 0.82)\end{array}$ & $\begin{array}{c}1.08 \\
(0.85 ; 1.38)\end{array}$ \\
\hline Body mass index $(\mathrm{kg} \cdot \mathrm{m}-2)$ & $p=0.87 \star *$ & $p=0.82 * \star$ & $p=0.68$ ** & $p=0.29 * *$ & $p=0.07 * \star$ & $p=0.93 * \star$ & $\mathrm{p}<0.001$ ** \\
\hline$<18.5$ & 1.00 & 1.00 & 1.00 & 1.00 & 1.00 & 1.00 & 1.00 \\
\hline $18.5-24.9$ & $\begin{array}{c}0.93 \\
(0.82 ; 1.05)\end{array}$ & $\begin{array}{c}1.08 \\
(0.87 ; 1.35)\end{array}$ & $\begin{array}{c}1.00 \\
(0.76 ; 1.32)\end{array}$ & $\begin{array}{c}1.06 \\
(0.80 ; 1.41)\end{array}$ & $\begin{array}{c}1.01 \\
(0.70 ; 1.44)\end{array}$ & $\begin{array}{c}0.96 \\
(0.63 ; 1.47)\end{array}$ & $\begin{array}{c}0.94 \\
(0.54 ; 1.63)\end{array}$ \\
\hline $25.0-29.9$ & $\begin{array}{c}0.93 \\
(0.82 ; 1.06)\end{array}$ & $\begin{array}{c}1.09 \\
(0.87 ; 1.37)\end{array}$ & $\begin{array}{c}0.98 \\
(0.75 ; 1.30)\end{array}$ & $\begin{array}{c}1.07 \\
(0.80 ; 1.41)\end{array}$ & $\begin{array}{c}0.89 \\
(0.61 ; 1.28)\end{array}$ & $\begin{array}{c}1.00 \\
(0.65 ; 1.54)\end{array}$ & $\begin{array}{c}1.13 \\
(0.65 ; 1.98)\end{array}$ \\
\hline$\geq 30.0$ & $\begin{array}{c}0.93 \\
(0.81 ; 1.07)\end{array}$ & $\begin{array}{c}1.04 \\
(0.82 ; 1.32)\end{array}$ & $\begin{array}{c}1.05 \\
(0.79 ; 1.40)\end{array}$ & $\begin{array}{c}0.96 \\
(0.71 ; 1.29)\end{array}$ & $\begin{array}{c}0.89 \\
(0.60 ; 1.30)\end{array}$ & $\begin{array}{c}0.95 \\
(0.60 ; 1.50)\end{array}$ & $\begin{array}{c}1.47 \\
(0.83 ; 2.60)\end{array}$ \\
\hline
\end{tabular}

(continues) 
Table 3 (continued)

\begin{tabular}{|c|c|c|c|c|c|c|c|}
\hline \multirow[t]{2}{*}{ Variables } & \multirow{2}{*}{$\begin{array}{l}\text { Healthy diet } \\
\text { PR }(95 \% \mathrm{Cl})\end{array}$} & \multirow{2}{*}{$\begin{array}{l}\text { Physical } \\
\text { activity } \\
\text { PR }(95 \% \mathrm{Cl})\end{array}$} & \multirow{2}{*}{$\begin{array}{l}\begin{array}{c}\text { Seeing a } \\
\text { doctor }\end{array} \\
\text { PR }(95 \% \mathrm{Cl})\end{array}$} & \multirow{2}{*}{$\begin{array}{l}\text { Not smoking } \\
\text { PR }(95 \% \mathrm{Cl})\end{array}$} & \multirow{2}{*}{$\begin{array}{l}\text { Not drinking } \\
\text { alcohol in } \\
\text { excess }\end{array}$} & \multirow{2}{*}{$\begin{array}{l}\begin{array}{c}\text { Avoiding } \\
\text { stress }\end{array} \\
\text { PR }(95 \% \mathrm{Cl})\end{array}$} & \multirow{2}{*}{$\begin{array}{l}\text { Keeping the } \\
\text { ideal weight } \\
\text { PR }(95 \% \mathrm{Cl})\end{array}$} \\
\hline & & & & & & & \\
\hline Smoking & $p<0.001$ * & $p=0.14$ * & $p=0.51 *$ & $p=0.02$ * & $p=0.12$ * & $p=0.03$ * & $p=0.26$ * \\
\hline Never smoked & 1.00 & 1.00 & 1.00 & 1.00 & 1.00 & 1.00 & 1.00 \\
\hline \multirow[t]{2}{*}{ Former smoker } & 0.91 & 0.94 & 0.97 & 1.10 & 1.14 & 1.22 & 0.93 \\
\hline & $(0.86 ; 0.97)$ & $(0.87 ; 1.02)$ & $(0.89 ; 1.06)$ & $(1.00 ; 1.21)$ & $(0.99 ; 1.31)$ & $(1.05 ; 1.41)$ & $(0.75 ; 1.14)$ \\
\hline \multirow[t]{2}{*}{ Current smoker } & 0.90 & 0.94 & 0.94 & 1.12 & 1.11 & 1.08 & 1.12 \\
\hline & $(0.86 ; 0.95)$ & $(0.88 ; 1.01)$ & $(0.85 ; 1.04)$ & $(1.03 ; 1.23)$ & $(0.97 ; 1.27)$ & $(0.93 ; 1.26)$ & $(0.92 ; 1.36)$ \\
\hline Physical activity level (min.wk-1) & $p=0.58 * \star$ & $p<0.001 \star \star$ & $p=0.02 * \star$ & $p=0.37^{\star \star}$ & $p=0.10^{\star \star}$ & $p=0.04^{\star \star}$ & $p=0.84 * \star$ \\
\hline 0 & 1.00 & 1.00 & 1.00 & 1.00 & 1.00 & 1.00 & 1.00 \\
\hline \multirow[t]{2}{*}{$10-149$} & 1.03 & 1.19 & 1.08 & 0.83 & 0.88 & 0.94 & 0.87 \\
\hline & $(0.98 ; 1.09)$ & $(1.10 ; 1.29)$ & $(0.97 ; 1.19)$ & $(0.72 ; 0.94)$ & $(0.74 ; 1.04)$ & $(0.79 ; 1.13)$ & $(0.68 ; 1.11)$ \\
\hline \multirow[t]{2}{*}{$\geq 150$} & 0.98 & 1.28 & 0.87 & 0.98 & 0.90 & 0.85 & 1.00 \\
\hline & $(0.93 ; 1.03)$ & $(1.20 ; 1.36)$ & $(0.79 ; 0.96)$ & $(0.89 ; 1.08)$ & $(0.79 ; 1.03)$ & $(0.74 ; 0.99)$ & $(0.83 ; 1.20)$ \\
\hline
\end{tabular}

PR: prevalence ratio; $95 \% \mathrm{Cl}$ : 95\% confidence interval.

* Wald test for heterogeneity;

** Wald test for trend.

that are not easily assessed 20,21 . In fact, our study shows that the health indicators chosen change according to several sociodemographic and behavioral variables.

Health professionals face a huge challenge trying to make individuals positively change their lifestyles. Because behavior is determined by a complex sets of factors, frequently poorly understood, many interventions aimed at changing some behaviors or attitudes have failed to do so, particularly in the long term 22,23 . The distribution of the target risk factors on the population and the population's knowledge and awareness of the harmful effects of these factors are examples of information needed to guide health interventions. Studies focusing on knowledge and awareness are emerging and the results indicate that individuals recognize the consequences of being exposed to risk factors 14,15 . Results from these studies are extremely important and intriguing because they show that many people are aware of risk factors, but do not change their lifestyles.

In 1997, a survey, including 15 members of the European Union also assessed awareness of health-related factors in terms of importance for health 17. In our study, we encouraged subjects to choose three factors (in order of importance), while in the European study subjects ranked only two factors. Similar results between our study and the European study 17 should be noted. Having a healthy diet was the most frequently mentioned factor in our sample, while "food" was second in
Europe. Overall, physical activity was the second most reported factor in our study. Although this finding indicates that people are recognizing physical activity as important for health, alarming rates of inactivity are observed in Brazil and worldwide 12,13,24. Therefore, one may consider that information on the benefits of physical activity and deleterious effects of inactivity are reaching the population. However, acquiring knowledge has not been enough to result in behavioral changes. Thus, to increase a population's levels of physical activity, besides disseminating healthrelated messages, structural improvements, such as providing easy access to appropriate facilities, must also be achieved 25. Recently, Yach et al. 26 released a report on how the example from tobacco control may aid policy makers to decrease the rates of inactivity and unhealthy habits. The authors discuss that smoking rates only began decreasing after numerous interventions, sustained advocacy, and policy expertise.

A major contribution of the present study to the literature is the evaluation of the variables associated with perceiving each factor. Several relevant differences were observed. In terms of sex, women cited healthy diet more frequently than men. The opposite was observed in terms of the health indicator physical activity. Compared to men, Brazilian women have healthier diets and lower levels of leisure-time physical activity 27,28, indicating coherence between these data. Nonetheless, somewhat surprising findings were also found. Older and poorer individuals reported the 
health indicators avoiding smoking and drinking in excess more frequently than their counterparts. On the other hand, richer individuals reported physical activity and healthy diet more frequently than the poorer. These findings suggest that messages about physical activity and healthy diets are mostly reaching the wealthy (and extended to the younger in terms of physical activity), while more established risk factors (i.e. smoking and drinking) are more perceived by poorer and older people. More interesting is the prevalence of smoking that was higher among poorer and older individuals than in richer and younger people, while the prevalence of physical activity was lower among the poorer and older (data not shown). These results summarize the complexity of the interrelationship between awareness on a specific health factor and the corresponding attitude towards it.
In summary, our study is one of the few to ask individuals to rank some well-known healthrelated factors in terms of importance for health. According to individuals' opinions, the most important factors for health are having a healthy diet and engaging in physical activity regularly. However, because unhealthy diets and physical inactivity are among the most prevalent risk factors for chronic diseases, interventions must focus on aspects other than merely increase knowledge about these factors. For example, because experiences in early life have been shown to exert long term effects on later behaviors 29,30 , interventions in childhood and adolescence are warranted. Furthermore, environmental aspects are considered important and influential over food choices consumers make and population leisure-time physical activity levels 31 and, therefore, must also be prioritized.

\section{Resumo}

O objetivo foi avaliar como adultos brasileiros ordenam sete fatores relacionados à saúde em termos de importância para a saúde. Estudo de base populacional realizado em Pelotas, Rio Grande do Sul, Brasil ( $N=3.100$; taxa de resposta: 96,5\%). Com base nos fatores listados, os indivíduos ordenaram três que, de acordo com sua opinião, fossem os mais importantes para a saúde. Os fatores investigados foram: "controlar o estresse", "praticar atividade física regularmente", "evitar beber em excesso", "evitar fumar", "consultar um médico regularmente", "manter o peso ideal" e "ter uma dieta saudá- vel". Dieta saudável (73,9\%), atividade física (59,9\%) $e$ visitar um médico $(45,7 \%)$ foram os mais citados. Indivíduos jovens e aqueles de melhor nível econômico relataram atividade física e estresse como fatores importantes para a saúde mais freqüentemente que seus pares. A importância atribuída para fatores relacionados à saúde altera-se drasticamente entre subgrupos populacionais.

Saúde do Adulto; Fatores de Risco; Dieta; Atividade Motora 


\section{Contributors}

F. F. Reichert participated in the planning of the research, data analysis and write up of the manuscript. M. R. Domingues, P. C. Hallal and A. J. D. Barros contributed with the planning of the study and reviews of the manuscript. M. R. Azevedo collaborated through reviewing the manuscript and statistical analyses. F. V. Siqueira participated in the data analysis and write up of the manuscript.

\section{Acknowledgments}

The authors acknowledge the Brazil Higher Education Consortia Program (CAPES) for supporting F. F. Reichert with an academic scholarship during the period of this project and for partially funding this study.

\section{References}

1. Doll R, Hill AB. Smoking and carcinoma of the lung; preliminary report. Br Med J 1950; 2:739-48.

2. Morris JN, Heady JA, Raffle PA, Roberts CG, Parks JW. Coronary heart-disease and physical activity of work. Lancet 1953; 265:1111-20.

3. Corrao G, Bagnardi V, Zambon A, La Vecchia C. A meta-analysis of alcohol consumption and the risk of 15 diseases. Prev Med 2004; 38:613-9.

4. Mooy JM, Vries H, Grootenhuis PA, Bouter LM, Heine RJ. Major stressful life events in relation to prevalence of undetected type 2 diabetes: the Hoorn Study. Diabetes Care 2000; 23:197-201.

5. Puddey IB, Rakic V, Dimmitt SB, Beilin LJ. Influence of pattern of drinking on cardiovascular disease and cardiovascular risk factors: a review. Addiction 1999; 94:649-63.

6. Wannamethee SG, Shaper AG, Whincup PH. Alcohol and adiposity: effects of quantity and type of drink and time relation with meals. Int J Obes (Lond) 2005; 29:1436-44.
7. World Health Organization. Global strategy on diet, physical activity and health. Geneva: World Health Organization; 2004.

8. Yusuf S, Hawken S, Ounpuu S, Dans T, Avezum A, Lanas F, et al. Effect of potentially modifiable risk factors associated with myocardial infarction in 52 countries (the INTERHEART study): case-control study. Lancet 2004; 364:937-52.

9. Menéndez Villalva C, Montes Martínez A, Núñez Losada C, Fernández Domínguez MJ, Gamarra Mondelo T, Buján Garmendia S. Estrés ambiental y reactividad cardiovascular: la influencia de los acontecimientos vitales estresantes en pacientes hipertensos. Aten Primaria 2002; 30:631-7.

10. Centers for Disease Control and Prevention. Cigarette smoking among adults: United States, 2002. MMWR Morb Mortal Wkly Rep 2004; 53:427-31. 
11. Costa JS, Silveira MF, Gazalle FK, Oliveira SS, Hallal PC, Menezes AM, et al. Heavy alcohol consumption and associated factors: a population-based study. Rev Saúde Pública 2004; 38:284-91.

12. Hallal PC, Victora CG, Wells JC, Lima RC. Physical inactivity: prevalence and associated variables in Brazilian adults. Med Sci Sports Exerc 2003; 35:1894-900.

13. Varo JJ, Martínez-González MA, De Irala-Estévez J, Kearney J, Gibney M, Martínez JA. Distribution and determinants of sedentary lifestyles in the European Union. Int J Epidemiol 2003; 32:138-46.

14. Ashley MJ, Cohen JE. What the public thinks about the tobacco industry and its products. Tob Control 2003; 12:396-400.

15. Domingues MR, Araújo CL, Gigante DP. Conhecimento e percepção sobre exercício físico em uma população adulta urbana do sul do Brasil. Cad Saúde Pública 2004; 20:204-15.

16. Morrow Jr. JR, Krzewinski-Malone JA, Jackson AW, Bungum TJ, FitzGerald SJ. American adults' knowledge of exercise recommendations. Res Q Exerc Sport 2004; 75:231-7.

17. Kafatos A, Manios Y, Markatji I, Giachetti I, Vaz de Almeida MD, Engstrom LM. Regional, demographic and national influences on attitudes and beliefs with regard to physical activity, body weight and health in a nationally representative sample in the European Union. Public Health Nutr 1999; 2:87-95.

18. Haskell WL, Lee IM, Pate RR, Powell KE, Blair SN, Franklin BA, et al. Physical activity and public health: updated recommendation for adults from the American College of Sports Medicine and the American Heart Association. Circulation 2007; 116:1081-93.

19. Barros AJ, Hirakata VN. Alternatives for logistic regression in cross-sectional studies: an empirical comparison of models that directly estimate the prevalence ratio. BMC Med Res Methodol 2003; 3:21.

20. Bault N, Coricelli G, Rustichini A. Interdependent utilities: how social ranking affects choice behavior. PLoS One 2008; 3:e3477.

21. Chin NP, Monroe A, Fiscella K. Social determinants of (un)healthy behaviors. Educ Health (Abingdon) 2000; 13:317-28.
22. Emmons KM, Linnan LA, Shadel WG, Marcus B, Abrams DB. The Working Healthy Project: a worksite health-promotion trial targeting physical activity, diet, and smoking. J Occup Environ Med 1999; 41:545-55.

23. Adams J, White M. Why don't stage-based activity promotion interventions work? Health Educ Res 2005; 20:237-43.

24. Balluz L, Ahluwalia IB, Murphy W, Mokdad A, Giles W, Harris VB. Surveillance for certain health behaviors among selected local areas: United States, Behavioral Risk Factor Surveillance System, 2002. MMWR Surveill Summ 2004; 53:1-100.

25. Trost SG, Owen N, Bauman AE, Sallis JF, Brown W. Correlates of adults' participation in physical activity: review and update. Med Sci Sports Exerc 2002; 34:1996-2001.

26. Yach D, McKee M, Lopez AD, Novotny T. Improving diet and physical activity: 12 lessons from controlling tobacco smoking. BMJ 2005; 330:898-900.

27. Bonomo E, Caiaffa WT, Cesar CC, Lopes ACS, Lima-Costa MF. Consumo alimentar da população adulta segundo perfil sócio-econômico e demográfico: Projeto Bambuí. Cad Saúde Pública 2003; 19: 1461-71.

28. Dias-da-Costa JS, Hallal PC, Wells JCK, Daltoé T, Fuchs SC, Menezes AMB, et al. Epidemiology of leisure-time physical activity: a population-based study in southern Brazil. Cad Saúde Pública 2005; 21:275-82.

29. Hallal PC, Wells JC, Reichert FF, Anselmi L, Victora CG. Early determinants of physical activity in adolescence: prospective birth cohort study. BMJ 2006; 332:1002-7.

30. Lake AA, Mathers JC, Rugg-Gunn AJ, Adamson AJ. Longitudinal change in food habits between adolescence (11-12 years) and adulthood (32-33 years): the ASH30 Study. J Public Health (Oxf) 2006; 28:10-6.

31. Popkin BM, Duffey K, Gordon-Larsen P. Environmental influences on food choice, physical activity and energy balance. Physiol Behav 2005; 86: 603-13.

Submitted on 23/Feb/2009

Final version resubmitted on $01 /$ Oct $/ 2009$

Approved on 18/Dec/2009 